\title{
Low-dose paclitaxel downregulates MYC proto-oncogene bHLH transcription factor expression in colorectal carcinoma cells
}

\author{
WENJING LI ${ }^{1 *}$, WANYUN ZHU²*, CHAOXIANG LV ${ }^{1}$, HAO QU ${ }^{1}$, KAIXIANG XU ${ }^{1}$, HONGHUI LI ${ }^{1}$, HAIFENG LI $^{2}$, \\ YIMING DU ${ }^{2}$, GUANGMING LIU ${ }^{2}$, YUNYUE WANG ${ }^{1}$, HONG-JIANG WEI ${ }^{1,3}$ and HONG-YE ZHAO ${ }^{1,2}$ \\ ${ }^{1}$ State Key Laboratory for Conservation and Utilization of Bio-Resources in Yunnan, Yunnan Agricultural University, \\ Kunming, Yunnan 650201; ${ }^{2}$ Pharmaceutical and Chemical Academy, Dali University, Dali, Yunnan 671003; \\ ${ }^{3}$ College of Animal Science and Technology, Yunnan Agricultural University, Kunming, Yunnan 650201, P.R. China
}

Received April 7, 2016; Accepted June 15, 2017

DOI: $10.3892 / \mathrm{ol} .2017 .7525$

\begin{abstract}
Paclitaxel (PTX) has been commonly used to treat multiple types of tumor. Its anticancer mechanism differs based on different PTX concentrations and types of tumor cell. In the present study, MTT assays of HCT116 and LOVO cells treated with PTX revealed the chemosensitivity of the cell lines for different PTX concentrations. The half-maximal inhibitory concentration values of PTX for these cells were 2.46 and $2.24 \mathrm{nM}$, respectively. Cell morphology observation revealed that both cell lines exhibited rounded, wrinkled and damaged morphologies with increasing concentrations of PTX. Fluorescence-activated cell sorting analysis indicated that $1 \mathrm{nM}$ PTX increased the proportion of cells in sub- $\mathrm{G}_{1}$ phases and decreased the proportion of cells in $G_{0} / G_{1}$ phases, whereas the proportions of cells in $\mathrm{S}$ and $\mathrm{G}_{2} / \mathrm{M}$ phases only slightly changed for both cell lines. Western blot analysis indicated that the total/nuclear protein expression of MYC proto-oncogene bHLH transcription factor (c-Myc) and phosphorylated (P)-c-Myc decreased in HCT116 cells in a dose-dependent manner, whereas the nuclear protein expression of P-c-Myc increased in LOVO cells in a dose-dependent manner. These results suggest that low-dose PTX downregulates c-Myc and P-c-Myc expression, subsequently inhibiting the cell cycle at $\mathrm{G}_{0} / \mathrm{G}_{1}$ in colorectal carcinoma.
\end{abstract}

Correspondence to: Professor Hong-Ye Zhao or Professor Hong-Jiang Wei, State Key Laboratory for Conservation and Utilization of Bio-Resources in Yunnan, Yunnan Agricultural University, 452 Fengyuan Road, Kunming, Yunnan 650201, P.R. China

E-mail: hyzhao2000@126.com

E-mail: hongjiangwei@126.com

*Contributed equally

Key words: paclitaxel, colorectal carcinoma cells, phosphorylated-MYC proto-oncogene bHLH transcription factor, cell cycle

\section{Introduction}

Paclitaxel (PTX), an antineoplastic drug, is commonly used as a first-line therapy for certain general types of malignancy, including lung, breast and ovarian cancer. Furthermore, low-dose PTX has been used to treat noncancer human diseases (1), and the anticancer activity of low concentrations of PTX has been investigated in specific tumor types (2-4). PTX causes cell cycle arrest and induces cell death in a concentration-dependent manner primarily by stabilizing polymerized microtubules, and enhancing microtubule assembly (5). PTX blocks $G_{0} / G_{1}$ phases or prevents $G_{2} / M$ phases of the cell cycle, causing cell death (6). The inhibitory effects of low-dose PTX on the metastasis and progress of cancer primarily depends on blocking angiogenesis and lymphangiogenesis (7). In addition, low-dose PTX has been demonstrated to induce the upregulation of thrombospondin-1 expression and downregulation of vascular endothelial growth factor expression in breast cancer (8). The findings of these previous studies suggest that determining the mechanism of a low concentration of PTX may aid in the effective application of PTX in clinical practice.

MYC proto-oncogene bHLH transcription factor (c-Myc), which belongs to the Myc gene family, is a pleiotropic transcription factor that participates in numerous cellular processes, including cell proliferation, apoptosis, differentiation, metabolism, genome stability and DNA repair (9). Thus far, $20 \%$ of human cancer types have been associated with c-Myc overexpression; c-Myc overexpression is frequently observed in breast and cervix carcinoma, small-cell lung cancer, osteosarcoma, and myeloid leukemia (10). Aberrant c-Myc expression is likely ascribable to direct gene alterations, which are associated with tumorigenesis and sustained tumor growth (11). Thus, the inhibition of c-Myc has promise as a therapeutic strategy for treating human cancer (12).

Colorectal carcinoma (CRC) is the third leading cause of cancer-associated mortalities worldwide (13). Despite advances in CRC diagnosis and treatment, 142,820 new CRC cases are diagnosed each year (14). Colorectal carcinogenesis is associated with genetic abnormalities; for example, elevated c-Myc expression has been identified in 44\% of CRCs (15). Therefore, manipulation of genetic abnormalities may be a promising approach for CRC treatment. 
The anticancer activity of low-dose PTX has been confirmed in certain types of cancer. However, no studies have investigated the effect of low-dose PTX on CRC cells, and no guidelines are available regarding the lowest effective concentrations of PTX for inhibiting the cell cycle. The aim of the present study was to evaluate whether low-dose PTX could downregulate the expression of c-Myc and phosphorylated (P)-c-Myc, thus inhibiting the cell cycle at the $\mathrm{G}_{0} / \mathrm{G}_{1}$ stage in CRC HCT116 and LOVO cells.

\section{Materials and methods}

Reagents and antibodies. PTX was purchased from Sigma-Aldrich (Merck KGaA, Darmstadt, Germany). Antibodies directed against c-Myc (cat. no. 1472-1), P-c-Myc (cat. no. 1203-1), $\beta$-actin (cat. no. P30002) and $\beta$-tubulin (cat. no. M30109) were obtained from Abcam (Cambridge, MA, USA). Antibody directed against poly(ADP-ribose) polymerase (PARP)-1 (cat. no. 2586S), were purchased from Santa Cruz Biotechnology, Inc. (Dallas, TX, USA). Horseradish peroxidase-conjugated goat anti-rabbit immunoglobulin (Ig)G and goat anti-mouse IgG antibodies (cat. nos. HAF007 and HAF008) were purchased from R\&D Systems, Inc. (Minneapolis, MN, USA). $\beta$-actin (cat. no. A5441) was purchased from Sigma-Aldrich; Merck KGaA. $\alpha$-tubulin was purchased from ProteinTech Group, Inc. (cat. no. 66031-1-lg; Chicago, IL, USA).

Cell lines and culture conditions. The cell lines LOVO, HCT116 and IEC-6 were purchased from Shanghai Cell Bank, Chinese Academy of Sciences (Shanghai, China). LOVO and HCT116 cells were cultured in RPMI-1640 (Gibco; Thermo Fisher Scientific, Inc., Waltham, MA, USA) and Dulbecco's modified Eagle's medium (DMEM)-F-12 (Hyclone; GE Healthcare Life Sciences, Logan, UT, USA) supplemented with $10 \%$ heat-inactivated fetal bovine serum (Gibco; Thermo Fisher Scientific, Inc.) and $100 \mathrm{IU} / \mathrm{ml}$ penicillin (Solarbio, Beijing, China), respectively. IEC-6 cells were maintained in DMEM medium (Gibco; Thermo Fisher Scientific, Inc.) supplemented with $10 \%$ heat-inactivated fetal bovine serum and $100 \mathrm{IU} / \mathrm{ml}$ penicillin. All cells were seeded in gelatin-coated $75-\mathrm{cm}^{2}$ flasks and cultured in $10 \mathrm{ml}$ of medium at $37^{\circ} \mathrm{C}$ in a humidified atmosphere of $5 \% \mathrm{CO}_{2}$ in air.

Cell morphology observations. The exponentially growing cells were transferred to 12 -well plates and cultured at $37^{\circ} \mathrm{C}$ in a $5 \% \mathrm{CO}_{2}$ atmosphere. HCT116 and LOVO cells were treated with 0,1 and $3 \mathrm{nM}$ PTX and then cultured at $37^{\circ} \mathrm{C}$ in a $5 \% \mathrm{CO}_{2}$ atmosphere for 3 days. Images were captured using an Olympus IX 71 microscope (magnification, x100; Olympus Corporation, Tokyo, Japan) when the cells reached $60-70 \%$ confluence.

MTT assay of cell survival. MTT colorimetric assay was used to determine the cytotoxicity of PTX. HCT116 and LOVO cells were plated in 96-well plates at densities of $1 \times 10^{3}$ and $2 \times 10^{3}$ cells/well, respectively, and were incubated with various concentrations of PTX (0.1-30 nM) for 3 days. Untreated cells were used as control groups. Then, $50 \mu \mathrm{l}$ of a $1 \mathrm{mg} / \mathrm{ml}$ solution of the MTT tetrazolium substrate (Sigma-Aldrich; Merck
Table I. Survival rate of colon cancer cells treated with PTX for 3 days.

\begin{tabular}{llc}
\hline & \multicolumn{2}{c}{ Survival, \% } \\
\cline { 2 - 3 } PTX $(\mathrm{nM})$ & HCT116 Cell & LOVO Cell \\
\hline 0.00 & $100.0 \pm 4.6593$ & $100.0 \pm 2.5036$ \\
0.03 & $84.78 \pm 2.4391$ & $97.56 \pm 4.7486$ \\
0.10 & $70.83 \pm 3.8203$ & $82.05 \pm 2.9887$ \\
0.30 & $66.22 \pm 4.3737$ & $68.59 \pm 3.3481$ \\
1.00 & $56.04 \pm 4.5880$ & $55.23 \pm 3.5017$ \\
3.00 & $38.20 \pm 4.0808$ & $41.54 \pm 4.9635$ \\
10.00 & $23.90 \pm 3.2255$ & $39.28 \pm 3.7560$ \\
30.00 & $19.08 \pm 1.1245$ & $27.37 \pm 4.4055$
\end{tabular}

Data are presented as the mean \pm standard deviation. PTX, paclitaxel.

$\mathrm{KGaA}$ ) in PBS was added to each well, and the plates were incubated for an additional $4 \mathrm{~h}$ at $37^{\circ} \mathrm{C}$. The resulting violet formazan precipitate was solubilized by the addition of $100 \mu \mathrm{l}$ of dimethyl sulfoxide (Sigma-Aldrich; Merck KGaA). All the plates were agitated for $5 \mathrm{~min}$ at room temperature and read immediately at $578 \mathrm{~nm}$ using a Bio-Rad Model 550 microplate reader (Bio-Rad Laboratories, Inc., Hercules, CA, USA). The half-maximal inhibitory concentration $\left(\mathrm{IC}_{50}\right)$ values of the examined compounds on different cell lines were obtained from the concentration-effect curves.

Flow cytometric analysis of the cell cycle. The cells were plated at densities of $3 \times 10^{4}$ cells/well in 6 -well plates and incubated for 1, 3 and 5 days with nutrient solution containing various concentrations of PTX extracts (0.1-30 nM). The cells were collected by centrifugation at $1,000 \mathrm{xg}$ for $5 \mathrm{~min}$ at $4^{\circ} \mathrm{C}$, fixed in cold $70 \%$ ethanol and stored at $-20^{\circ} \mathrm{C}$. The cells were subsequently washed with PBS, resuspended in cold PBS, and incubated with $10 \mathrm{mg} / \mathrm{ml}$ RNase and $1 \mathrm{mg} / \mathrm{ml}$ propidium iodide (Sigma-Aldrich; Merck KGaA) at $37^{\circ} \mathrm{C}$ for $30 \mathrm{~min}$. Flow cytometric analysis of DNA content was performed using a flow cytometer (BD Biosciences, San Jose, CA, USA). The percentages of cells in the different cell cycle phases were determined using FlowJo software version 9.3.2 (BD Biosciences, San Jose, CA, USA).

Protein extraction and immunoblotting. Subsequent to treatment of the LOVO and HCT116 cells with PTX at the indicated concentrations and times, the cells were washed twice with PBS and collected by centrifugation at $200 \mathrm{x} g$ for $5 \mathrm{~min}$ at $4^{\circ} \mathrm{C}$. Then, total/nuclear protein concentrations of cell lysates were determined using a BCA Protein Assay kit (Beyotime Institute of Biotechnology, Shanghai, China). Protein samples (total protein, $150 \mu \mathrm{g}$; nuclear protein, $50 \mu \mathrm{g}$ ) were separated using SDS-PAGE (10\% gel) and transferred onto polyvinylidene difluoride membranes. The membranes were incubated in 5\% bovine serum albumin buffer (Beijing Solarbio Science \& Technology Co., Ltd., Beijing, China) for $30 \mathrm{~min}$ at room temperature with gentle agitation to block nonspecific binding prior to incubation with the diluted primary antibody 


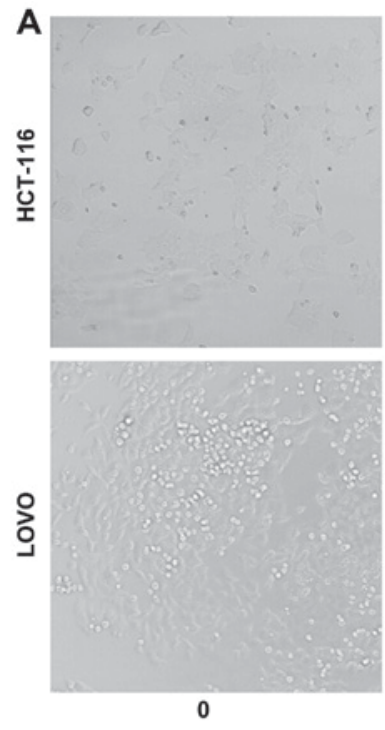

B

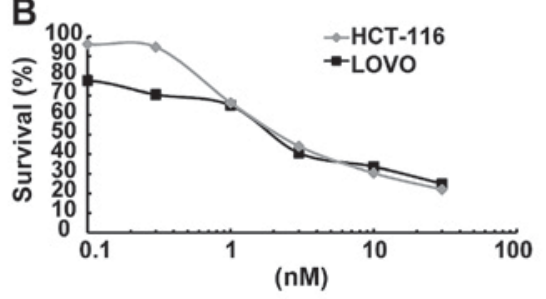

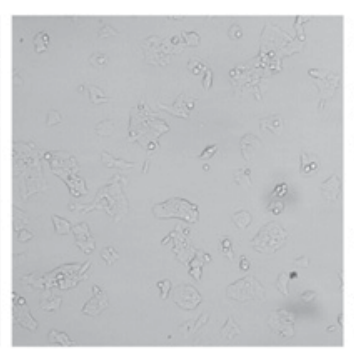
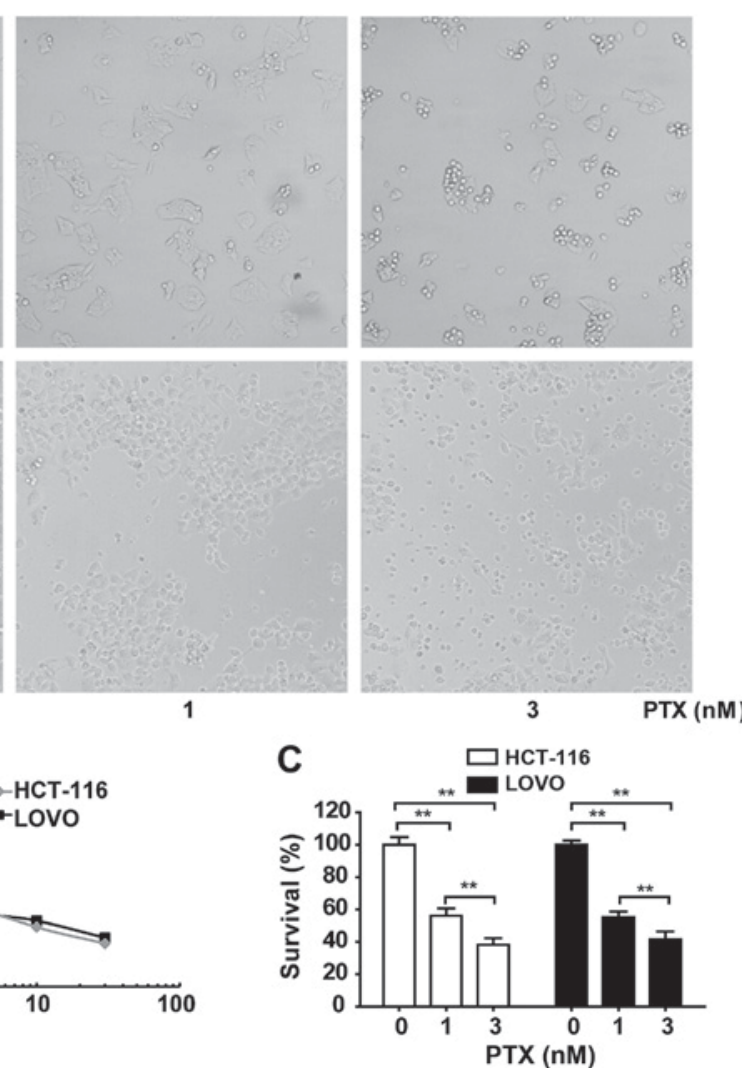

Figure 1. Chemosensitivity of colon cancer cells to different concentrations of PTX. (A) Images of HCT116 and LOVO cells exposed to the indicated concentrations of PTX for 3 days (original magnification, x100). (B) HCT116 and LOVO cells were exposed to 0.1, 0.3, 1, 3, 10 and 30 nM PTX for 3 days, and cell viability was determined by MTT assay using a spectrophotometer at $580 \mathrm{~nm}$. (C) Quantification of cell viability. Data are presented as the mean \pm standard deviation. ${ }^{* *} \mathrm{P}<0.01$ vs. control. PTX, paclitaxel.

(anti-c-Myc, 1:1,000; Abcam; anti-P-c-Myc, 1:500; Abcam; anti-PARP-1, 1:200; Santa Cruz Biotechnology) overnight at $4^{\circ} \mathrm{C}$. Subsequently, the membranes were incubated with diluted anti-rabbit or anti-mouse secondary antibody (1:5,000; R\&D Systems, Inc.) for $90 \mathrm{~min}$ at room temperature. The membranes were washed three times in PBS for 10 min each at room temperature and then the membranes were developed using the ECL detection system (EasySee Western Blot kit; Transgene SA, Strasbourg, France) and visualized with an Imaging lab ${ }^{\mathrm{TM}}$ software version 4.0 (Bio-Rad Laboratories, Inc.).

Statistical analysis. Statistical comparisons were performed using one-way analysis of variance followed by Scheffe's post hoc test using SPSS 22.0 software (IBM Corp., Armonk, NY, USA). Quantitative data are presented as the mean of triplicate experiments \pm standard deviation. $\mathrm{P}<0.05$ was considered to indicate a statistically significant difference.

\section{Results}

Effect of PTX treatment on cell viability. As high concentrations of PTX exhibit high cytotoxicity, the concentration that does not induce significant cell death needed to be determined. This determination was particularly important as cellular toxicity may interfere significantly with cellular signaling outcomes and gene expression. Therefore, cells were incubated for 3 days with PTX concentrations ranging from 0.1 to $30 \mathrm{nM}$, at which point MTT assays were performed (Fig. 1). The survival rates of HCT116 and LOVO cells indicated dose-dependent toxic effects of PTX on these cells (Table I). The $\mathrm{IC}_{50}$ values of PTX for HCT116 and LOVO cell lines were 2.46 and 2.24 nM, respectively (Fig. 1B). Cell viability reduced dose-dependently by 1 and 3 nM PTX (Fig. 1C). Cell morphology observations revealed that the CRC cell lines HCT116 and LOVO exhibited sensitivity to 1 , and $3 \mathrm{nM}$ PTX as indicated by significant decreases in cell survival compared with the control (Fig. 1C). Subsequently, two concentrations close to the $\mathrm{IC}_{50}$ values were used to treat these cells and it was observed that cell lines exhibited rounded, wrinkled and damaged morphologies with increasing concentrations of PTX (Fig. 1A).

Effects of different concentrations PTX on the cell cycle distribution of CRC cells. As is known, PTX can induce $\mathrm{G}_{2} / \mathrm{M}$ cell cycle arrest in breast cancer (16). To determine whether PTX has an effect on the cell cycle distribution of colon cancer cells, flow cytometric analysis on the cell cycle was performed. After the cells were treated with PTX $(1 \mathrm{nM})$ for 1,3 or 5 days, the proportion of HCT116 and LOVO cells in the sub- $\mathrm{G}_{1}$ phases increased and the proportion of these cells in $G_{0} / G_{1}$ phases decreased, whereas the proportions of cells in $S$ and $G_{2} / M$ phases only slightly changed for both cell lines (Fig. 2).

PTX regulates the total protein expression of $c-M y c$ and $P-c-M y c$ in CRC cells. c-Myc drives cell cycle progression and is extensively controlled through post-translational modifications, with a major role for phosphorylated c-Myc. 
A

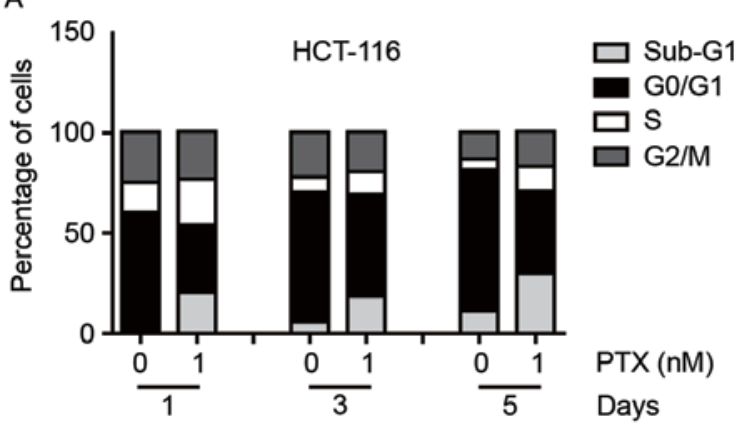

B

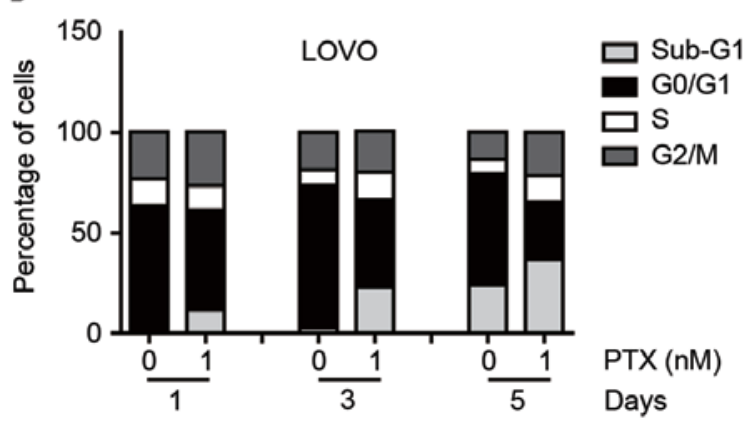

Figure 2. Effect of PTX on the cell cycle distribution of colon cancer cells. Histogram of the cell cycle distribution of (A) HCT116 and (B) LOVO cells treated with PTX. HCT116 and LOVO cells were exposed to 0 and $1 \mathrm{nM}$ PTX for 1, 3 and 5 days, and then cell cycle distribution was detected using flow cytometric analysis. PTX, paclitaxel.

A

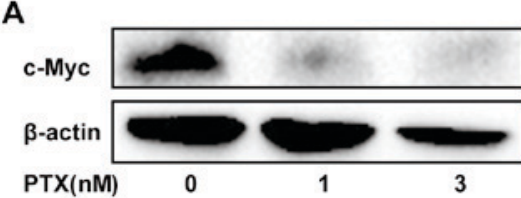

C

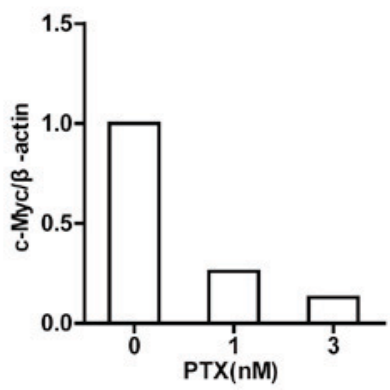

E

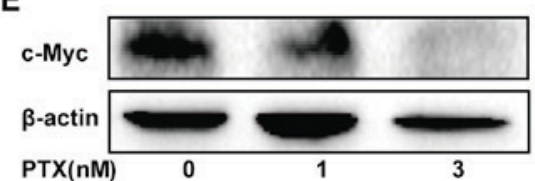

G

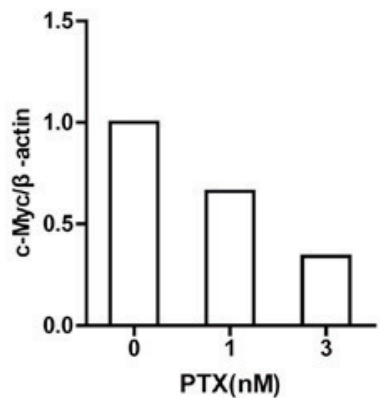

B

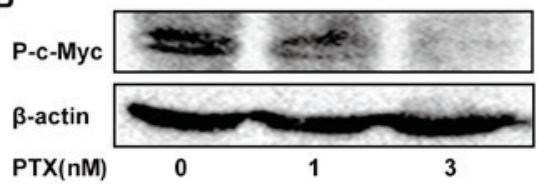

D

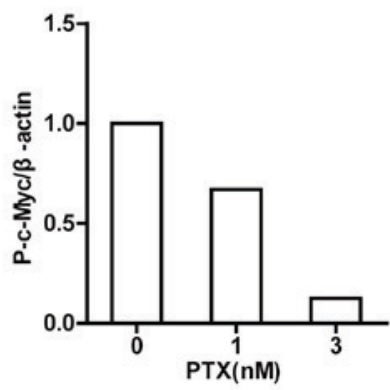

F

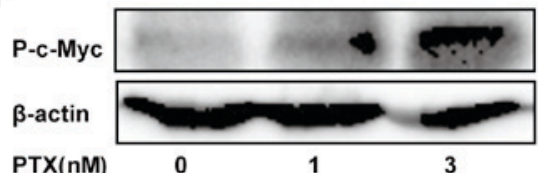

H

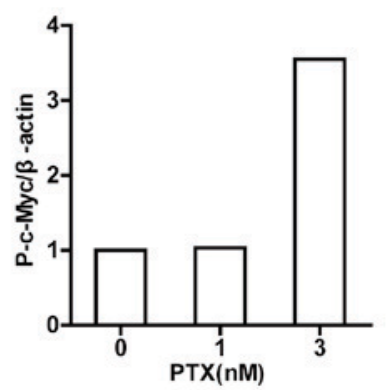

Figure 3. Total protein expression of c-Myc and P-c-Myc in colon cancer cell lines. HCT116 and LOVO cells were treated with PTX at the indicated concentrations for 3 days. Whole cell extracts were prepared, and equal amounts of protein were separated by SDS-PAGE. $\beta$-actin was used as a control for protein loading. Protein expression levels were examined using western blot analysis. Representative blot of the total protein expression of (A) c-Myc and (B) P-c-Myc in HCT116 cells. Quantification of the total protein expression of (C) c-Myc and (D) P-c-Myc in HCT116 cells. Representative blot of the total protein expression of (E) c-Myc and (F) P-c-Myc in LOVO cells. Quantification of the total protein expression of (G) c-Myc and (H) P-c-Myc in LOVO cells. PTX, paclitaxel; $\mathrm{P}-$, phosphorylated; c-Myc, MYC proto-oncogene bHLH transcription factor.

c-Myc phosphorylation has been associated with protein stabilization and described to occur as cells enter mitosis (17). Thus, western blot analysis was performed to determine the total protein expression levels of c-Myc and P-c-Myc (Fig. 3).
PTX treatment of HCT116 cells induced dose-dependent decreases in c-Myc and P-c-Myc total protein expression levels (Fig. 3A-D). In LOVO cells, PTX treatment induced a dose-dependent decrease in c-Myc total protein expression, 
A

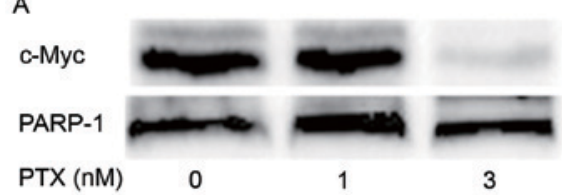

C

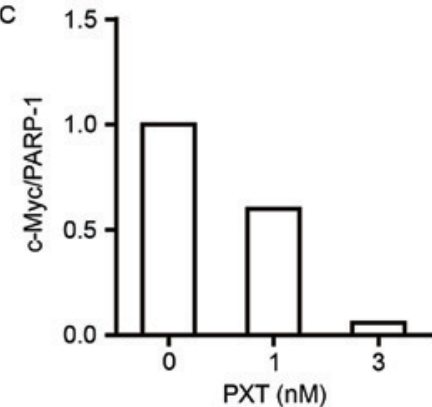

E

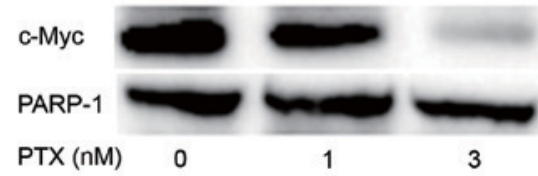

G

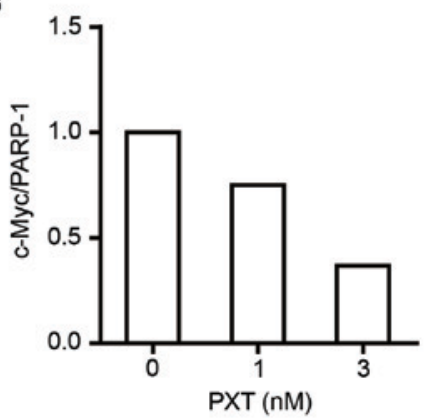

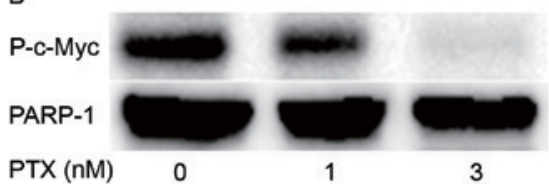
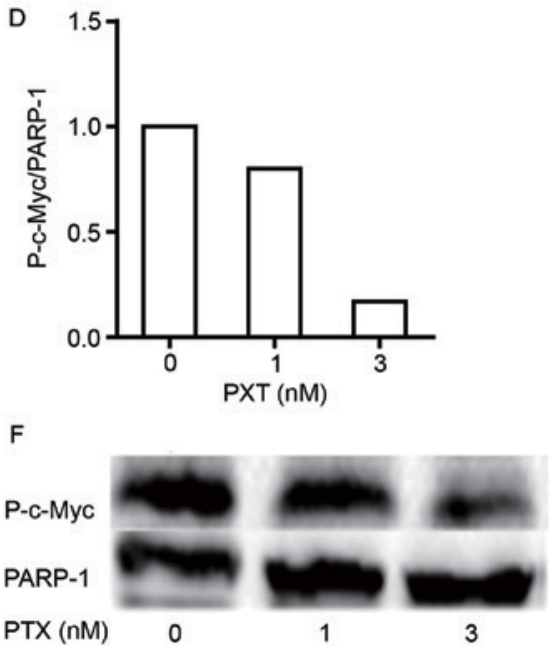

$\mathrm{H}$

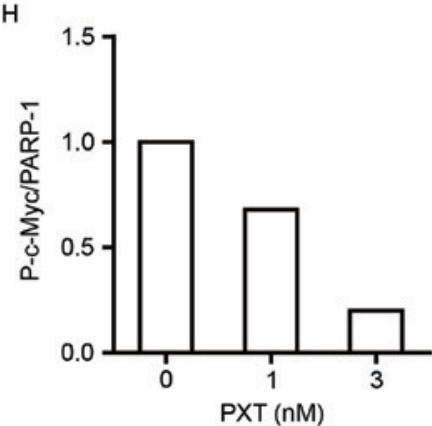

Figure 4. Nuclear protein expression of c-Myc and P-c-Myc in colon cancer cells. In total, $50 \mu \mathrm{g}$ of nuclear protein was electrophoretically separated on a $10 \%$ SDS gel, immunoblotted and hybridized with specific antibodies. HCT116 and LOVO cells were treated with the same conditions as aforementioned. Representative blot of nuclear the nuclear protein expression of (A) c-Myc and (B) P-c-Myc in HCT116 cells. Quantification of the nuclear protein expression of (C) c-Myc and (D) P-c-Myc in HCT116 cells. Representative blot of the nuclear protein expression of (E) c-Myc and (F) P-c-Myc in LOVO cells. Quantification of the nuclear protein expression of $(\mathrm{G}) \mathrm{c}-\mathrm{Myc}$ and $(\mathrm{H})$ P-c-Myc in LOVO cells. PTX, paclitaxel; P-, phosphorylated; PARP, poly(ADP-ribose) polymerase; c-Myc, MYC proto-oncogene bHLH transcription factor.

A

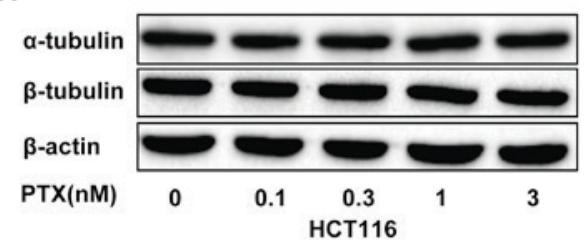

B

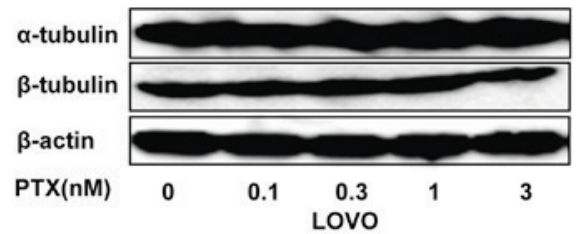

Figure 5. Effect of different concentrations of PTX on the protein expression of $\alpha$-tubulin and $\beta$-tubulin in colon cancer cell lines. HCT116 and LOVO cells were treated as aforementioned, whole cell extracts were prepared, and equal amounts of protein were separated by SDS-PAGE followed by immunoblotting to determine the protein expression of $\alpha$-tubulin and $\beta$-tubulin in (A) HCT116 and (B) LOVO cells. $\beta$-actin was used as a loading control. PTX, paclitaxel. but a dose-dependent increase in P-c-Myc total protein expression (Fig. 3E-H).

PTX regulates the nuclear protein expression of $c-M y c$ and $P-c-M y c$ in CRC cells. c-Myc protein is regulated primarily by its sequestration in nucleoli; its phosphorylated form accumulates in the nuclei of tumor cells because of impaired ubiquitination by proteasomes (18). Thus, the nuclear protein expression of c-Myc and P-c-Myc in colon cancer cells treated with the indicated concentrations of PTX was detected. It was observed that the nuclear protein expression levels of c-Myc and P-c-Myc were decreased by PTX in a dose-dependent manner in HCT116 cells (Fig. 4A-D). The nuclear protein expression levels of c-Myc and P-c-Myc were also decreased in LOVO cells following PTX treatment (Fig. 4E-H).

PTX regulates the expression of $\alpha$-tubulin and $\beta$-tubulin in $C R C$ cells. The anticancer activity of PTX primarily depends on stabilizing polymerized microtubules and enhancing 


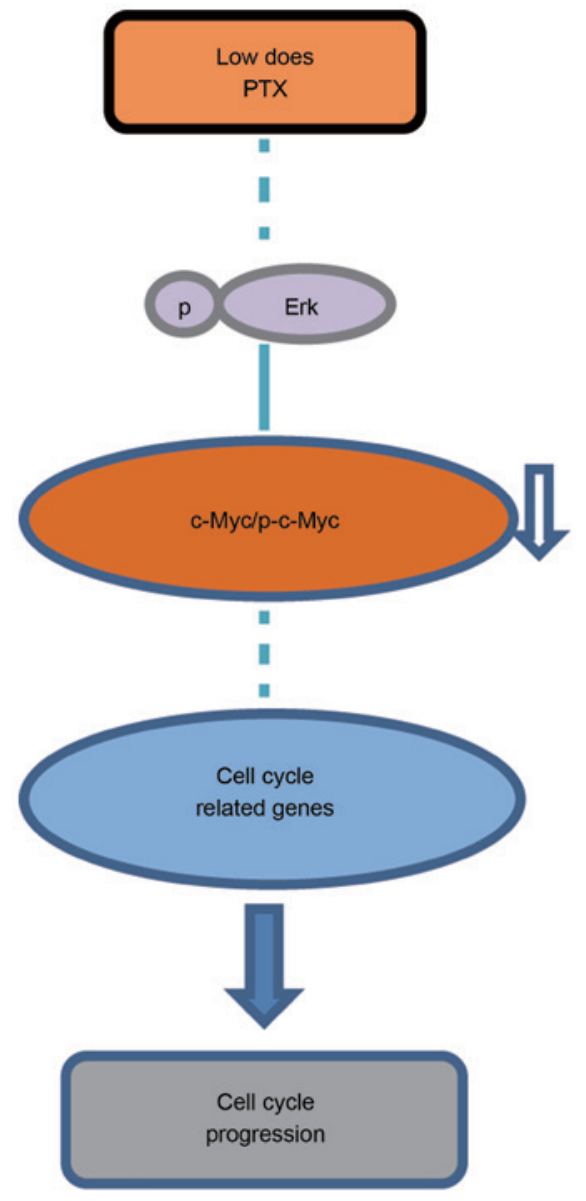

Figure 6. Schematic pathway for PTX regulation of the cell cycle in CRC cells. In this model, the downregulation of c-Myc and P-c-Myc by PTX in an unknown pathway results, which causes arrest of $\mathrm{G}_{0} / \mathrm{G}_{1}$ phases of the cell cycle. The blocking of $\mathrm{G}_{0} / \mathrm{G}_{1}$ phases may be involved with the activity of cyclin E/Cdk2 and cyclin D/Cdk4/6. PTX, paclitaxel; p, phosphorylated; Erk, extracellular regulated kinase; Cdk, cyclin dependent kinase; c-Myc, MYC proto-oncogene bHLH transcription factor.

microtubule assembly. Tubulin is a microtubule protein, the integrity of which is essential for the separation and segregation of chromosomes during cell division. High expression of tubulin reduces the chemosensitivity of numerous cancer types to PTX (19). To determine whether low-dose PTX affected the expression of $\alpha$-tubulin and $\beta$-tubulin in HCT116, and LOVO cells, these cells were treated with PTX at the indicated concentrations and times, and $\alpha$-tubulin and $\beta$-tubulin expression was examined. It was revealed that $\alpha$-tubulin and $\beta$-tubulin expression did not differ in these cells following PTX treatment (Fig. 5). A schematic representation of the proposed mechanism of cell cycle regulation by PTX in the CRC cell lines analyzed in the present study is demonstrated in Fig. 6.

\section{Discussion}

PTX is one of the most effective cytotoxic agents for the clinical treatment of cancer. However, its clinical benefit is often limited by dose-dependent toxicity and drug resistance. PTX generates antitumor activity by inhibiting cell proliferation and inducing cell apoptosis, with unavoidable damage to normal cells, severe anaphylactic hypersensitivity reactions, and peripheral neuropathy (20). Nevertheless, low-dose PTX halts the progress and metastasis of cancer through its antiangiogenic activities rather than by inducing tumor cell apoptosis (6). A previous study reported that low-dose PTX induced $\mathrm{G}_{0} / \mathrm{G}_{1}$ cell cycle arrest in esophageal squamous cell carcinoma larynx carcinoma and ovarian cancer (21). Consistently, the results of the present study demonstrated that $1 \mathrm{nM}$ PTX blocked $\mathrm{G}_{0} / \mathrm{G}_{1}$ phases of the cell cycle, and induced minimal apoptosis with less toxic effects in HCT116 and LOVO cells.

Tubulins serve essential roles in the chemosensitivity of cancer; their structural alterations could specifically modify PTX sensitivity in vitro, and high tubulin expression reduces the chemosensitivity of numerous types of cancer to PTX (22). The overexpression of tubulins has been reported in several types of cancer cells including breast cancer and acute lymphoblastic leukemia with PTX resistance (19). In the current study, the response of CRC cells to low-dose PTX was investigated. It was demonstrated that $0-0.3 \mathrm{nM}$ PTX exhibited no significant influence on the viability of HCT116 and LOVO cells, whereas 1-30 nM PTX significantly affected cell viability, the $\mathrm{IC}_{50}$ values of PTX for these cell lines were 2.46 and $2.24 \mathrm{nM}$, respectively. These results demonstrated that both cell lines have high chemosensitivity towards low-dose PTX. It was also demonstrated that $\alpha$-tubulin and $\beta$-tubulin expression did not differ in HCT116, and LOVO cells. These results suggest that low-dose PTX does not affect the structure of tubulin proteins in these cells.

A previous study has suggested that the use of PTX in antitumor therapeutic strategies should be rationally based on the molecular profile of the individual tumor by specifically analyzing Myc expression levels (23). Other anticancer drugs, including fluorouracil and niclosamide, have been reported to directly downregulate c-Myc expression in human colon cancer KM12C cells and human osteosarcoma cells, respectively $(24,25)$. PTX treatment of HT29-4D colon carcinoma cells, HL-60 promyelocytic leukemic cells and ovarian cancer cells has been suggested to indirectly downregulate c-Myc expression (26-28). In the present study, it was demonstrated that PTX treatment dose-dependently decreased the total/nuclear protein expression of c-Myc in both cell lines, the total/nuclear protein expression of P-c-Myc in HCT116 cells and the nuclear protein expression of P-c-Myc in LOVO cells. In contrast, in LOVO cells, low-dose PXT treatment dose-dependently increased the total protein expression of P-c-Myc. In a previous study, PTX treatment induced c-Myc and P-c-Myc redistribution in prostate carcinoma cell lines; these proteins underwent reorganization, and were more homogeneously diffused (29). Whether CRC cell lines have different responses to PTX treatment is unknown and requires further study.

The functions of c-Myc and P-c-Myc in cell growth, and transformation have been investigated (29); extracellular regulated kinase 2 has been revealed to phosphorylate c-Myc at threonine 58 (Thr58) and serine (Ser62), and to stimulate the activity of cyclin E/cyclin-dependent kinase 2 complexes (30). Janus kinase phosphorylates c-Myc at Ser62 and Ser71, which are associated with cell proliferation, and cell cycle regulation (31). The anti-P-c-Myc antibody used in the present study only detects c-Myc phosphorylated on Thr58 and Ser62. In conclusion, the findings of the current study demonstrated that PTX affects c-Myc through downregulating the expression of c-Myc and P-c-Myc in CRC cells. A greater understanding of 
the mechanisms by which PTX regulates the cell cycle may provide novel approaches for the treatment of CRC.

\section{Acknowledgements}

The present study was supported by the National Natural Science Foundation of China (grant nos. 81160282 and 31360549), Major Program on Basic Research Projects of Yunnan Province (grant no. 2014FC006), the Science Foundation Key Project of Yunnan Province Department of Education (grant no. ZD2013003), and Talent Project of Young and Middle-aged Academic Technology Leadership in Yunnan Province (grant no. 2013HB073).

\section{References}

1. Zhang D, Yang R, Wang S and Dong Z: Paclitaxel: New uses for an old drug. Drug Des Devel Ther 8: 279-284, 2014.

2. Hirose A, Tajima H, Ohta T, Tsukada T, Okamoto K, Nakanuma S, Sakai S, Kinoshita J, Makino I, Furukawa H, et al: Low dose paclitaxel inhibits the induction of epidermal-mesenchymal transition in the human cholangiocarcinoma CCKS-1 cell line. Oncol Lett 6: 915-920, 2013.

3. He Q, Li J, Yin W, Song Z, Zhang Z, Yi T, Tang J, Wu D, Lu Y, Wang Z, et al: Low-dose paclitaxel enhances the anti-tumor efficacy of GM-CSF surface-modified whole-tumor-cell vaccine in mouse model of prostate cancer. Cancer Immunol Immunother 60: 715-730, 2011.

4. Tsukada T, Fushida S, Harada S, Terai S, Yagi Y, Kinoshita J, Oyama K, Tajima H, Ninomiya I, Fujimura T and Ohta T: Low-dose paclitaxel modulates tumour fibrosis in gastric cancer. Int J Oncol 42: 1167-1174, 2013.

5. Barbuti AM and Chen ZS: Paclitaxel through the ages of anticancer therapy: Exploring its role in chemoresistance and radiation therapy. Cancer (Basel) 7: 2360-2371, 2015.

6. Bocci G, Di Paolo A and Danesi R: The pharmacological bases of the antiangiogenic activity of paclitaxel. Angiogenesis 16: 481-492, 2013.

7. Tao WY, Liang XS, Liu Y, Wang CY and Pang D: Decrease of let-7f in low-dose metronomic paclitaxel chemotherapy contributed to upregulation of thrombospondin-1 in breast cancer. Int $\mathrm{J}$ Biol Sci 11: 48-58, 2015.

8. Di Paolo A, Bocci G and Danesi R: The preclinical bases of the rational combination of paclitaxel and antiangiogenic drugs. Clin Cancer Drugs 1: 100-115, 2014.

9. Meyer N and Penn LZ: Reflecting on 25 years with MYC. Nat Rev Cancer 8: 976-990, 2008.

10. Pelengaris S, Khan M and Evan G: C-MYC: More than just a matter of life and death. Nat Rev Cancer 2: 764-776, 2002.

11. Chen BJ, Wu YL, Tanaka Y and Zhang W: Small molecules targeting c-Myc oncogene: Promising anti-cancer therapeutics. Int J Biol Sci 10: 1084-1096, 2014.

12. Zhang X, Zhao X, Fiskus W, Lin J, Lwin T, Rao R, Zhang Y, Chan JC, Fu K, Marquez VE, et al: Coordinated silencing of MYC-mediated miR-29 by HDAC3 and EZH2 as a therapeutic target of histone modification in aggressive B-cell lymphomas. Cancer Cell 22: 506-523, 2012.

13. Douaiher J, Ravipati A, Grama B, Chowdhury S, Alatise O and Are C: Colorectal cancer-global burden, trends, and geographical variations. J Surg Oncol 115: 619-630, 2017.

14. Torre LA, Bray F, Siegel RL, Ferlay J, Lortet-Tieulent J and Jemal A: Global cancer statistics, 2012. CA Cancer J Clin 65: 87-108, 2015

15. Goel A and Boland CR: Epigenetics of colorectal cancer. Gastroenterology 143: 1442-1460, 2012.

16. Li JP, Yang YX, Liu QL, Pan ST, He ZX, Zhang X, Yang T, Chen XW, Wang D, Qiu JX and Zhou SF: The investigational Aurora kinase A inhibitor alisertib (MLN8237) induces cell cycle $\mathrm{G} 2 / \mathrm{M}$ arrest, apoptosis, and autophagy via p38 MAPK and Akt/mTOR signaling pathways in human breast cancer cells Drug Des Devel Ther 9: 1627-1652, 2015.
17. Bottone MG, Soldani C, Tognon G, Gorrini C, Lazzè MC, Brison O, Ciomei M, Pellicciari C and Scovassi AI: Multiple effects of paclitaxel are modulated by a high c-myc amplification level. Exp Cell Res 290: 49-59, 2003.

18. Sanders JA and Gruppuso PA: Nucleolar localization of hepatic c-Myc: A potential mechanism for c-Myc regulation. Biochim Biophys Acta 1743: 141-150, 2005.

19. Xie S, Ogden A, Aneja R and Zhou J: Microtubule-binding proteins as promising biomarkers of Paclitaxel sensitivity in cancer chemotherapy. Med Res Rev 36: 300-312, 2016.

20. Flatters SJ, Xiao WH and Bennett GJ: Acetyl-L-carnitine prevents and reduces paclitaxel-induced painful peripheral neuropathy. Neurosci Lett 397: 219-223, 2006.

21. Yun T, Liu Y, Gao D, Linghu E, Brock MV, Yin D, Zhan Q, Herman JG and Guo M: Methylation of CHFR sensitizes esophageal squamous cell cancer to docetaxel and paclitaxel. Genes Cancer 6: 38-48, 2015.

22. Wang S, Qiu J, Shi Z, Wang Y and Chen M: Nanoscale drug delivery for taxanes based on the mechanism of multidrug resistance of cancer. Biotechnol Adv 33: 224-241, 2015.

23. Gatti G, Maresca G, Natoli M, Florenzano F, Nicolin A, Felsani A and D'Agnano I: MYC prevents apoptosis and enhances endoreduplication induced by paclitaxel. PLoS One 4: e5442, 2009.

24. Zhao HY, Ooyama A, Yamamoto M, Ikeda R, Haraguchi M, Tabata S, Furukawa T, Che XF, Iwashita K, Oka T, et al: Down regulation of c-Myc and induction of an angiogenesis inhibitor, thrombospondin-1, by 5-FU in human colon cancer KM12C cells. Cancer Lett 270: 156-163, 2008.

25. Liao Z, Nan G, Yan Z, Zeng L, Deng Y, Ye J, Zhang Z, Qiao M, Li R, Denduluri S, et al: The anthelmintic drug Niclosamide inhibits the proliferative activity of human osteosarcoma cells by targeting multiple signal pathways. Curr Cancer Drug Targets 15: 726-738, 2015.

26. Wilmes A, Chan A, Rawson P, William Jordan T and Miller JH: Paclitaxel effects on the proteome of HL-60 promyelocytic leukemic cells: Comparison to peloruside A. Invest New Drugs 30: 121-129, 2012.

27. el Khyari S, Bourgarel V, Barra Y, Braguer D and Briand C: Pretreatment by tubulin agents decreases C-MYC induction in human colon carcinoma cell line HT29-D4. Biochem Biophys Res Commun 231: 751-754, 1997.

28. Fu Q, Chen Z, Gong X, Cai Y, Chen Y, Ma X, Zhu R and Jin J: $\beta$-Catenin expression is regulated by an IRES-dependent mechanism and stimulated by paclitaxel in human ovarian cancer cells. Biochem Biophys Res Commun 461: 21-27, 2015.

29. Supino R, Favini E, Cuccuru G, Zunino F and Scovassi AI: Effect of paclitaxel on intracellular localization of c-Myc and P-c-Myc in prostate carcinoma cell lines. Ann N Y Acad Sci 1095: 175-181, 2007.

30. Yang W, Zheng Y, Xia Y, Ji H, Chen X, Guo F, Lyssiotis CA, Aldape K, Cantley LC and Lu Z: ERK1/2-dependent phosphorylation and nuclear translocation of PKM2 promotes the Warburg effect. Nat Cell Biol 14: 1295-1304, 2012.

31. Supino R and Scovassi AI: c-myc: A double-headed Janus that regulates cell survival and death. Gene Ther Mol Biol 8: 385-394, 2004. 